\section{Military Technical College Kobry El-Kobbah, Cairo, Egypt}

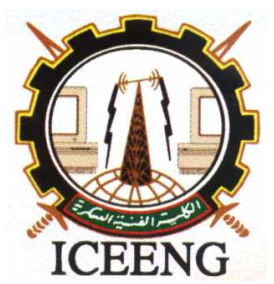

\author{
$6^{\text {th }}$ International Conference \\ on Electrical Engineering \\ ICEENG 2008
}

\title{
Security evaluation of VoIP cryptographic algorithms
}

By

Faiz Yousif Mohmmed*

Alaa Eldin Rohiem**

Ashraf Diaa Elbayoumy**

\section{$\underline{\text { Abstract: }}$}

The communications world is moving toward VoIP but does not have the security expertise it needs in-house to meet the real world stress it will encounter. Unfortunately adding security has a negative effect on the voice quality of service (QoS). Many researches study the effects of adding security to VoIP using different cryptographic algorithms by comparing end to end delay, jitter and packet loss. Subjective and objective test methods are used to measure QoS $[3,10]$. But in our paper a new point of view is included, the goal of the paper is to compare the powerful of the securing algorithm by measuring the randomness of its encrypted output. In addition we measure execution time (delay) to be another factor of comparison. A $\mathrm{C}++$ simulation program was written to simulate secure VoIP system. This paper organized as follows: 1 . Introduction Section present the VoIP motivation, challenges, and security issues, section 2. Explain VOIP model, section 3. Describe statistical test suite and test bed environment, section 4. Demonstrate the statistical results, and the last section Express the conclusion.

\section{Keywords:}

VoIP, SVoIP, Cryptography, StsGui, ASCII Converter, Randomness measurement, StsGui, authentication.

* Sudanese Armed Forces

** Egyptian Armed Forces 


\section{Introduction:}

Voice over IP (VoIP) can be briefly defined as the technology that allows the use of the IP protocol(s) to carry voice signalling and media traffic [1].

VoIP sound is sampled, quantified, digitized and compressed with an appropriate codec and streamed over traditional network architectures. Then, several coded speech frames are packetized to form the payload part of a packet (e.g. RTP packet). The headers (e.g. IP/UDP/RTP) are added to the payload and form a packet which is sent to IP networks. The packet may suffer different network impairments (e.g. packet loss, delay and jitter) in IP networks. It is and it behaves as normal IP data but at the same time has to obey to the rules imposed by classical telephony in terms of quality of service and availability $[2,3]$.

The main motivations for VoIP are: (bypass toll switches and save on call costs, Rich media conferencing combines voice, video, and data, mobility).VoIP service requirements are summarized in minimizing latency, enable bandwidth priority, ensuring reliability and ensuring security.

Confidentiality, Integrity, and Availability three key principles that should be guaranteed in any kind of secure system. This principle is applicable across the whole security spectrum. Confidentiality refers to mechanisms that ensure that only authorized individuals may access secure information. Cryptography and Encryption are examples of methods used to ensure confidentiality of data. Integrity means that information is unchanged as it moves between endpoints. Availability characterizes the operational state of the network [5].

VoIP data is transmitted in digital packet form. This means that the voice transmissions can be attacked, hacked, intercepted, manipulated, rerouted and degraded just as any data packet on the data network. Viruses, worms, trojan horses, denial of service attacks and hijacking are all possibilities on the VoIP network [4].

The implementation of various security measures can degrade QoS. These complications range from delaying or blocking of call setups by firewalls to encryption produced latency and delay variation (jitter) [5].

VOIP calls must achieve the $150 \mathrm{~ms}$ bound to successfully emulate the QoS that today's phones provide [6].

Adding security constraints significantly increases the bandwidth usage, causing more latency and jitter, thereby degrading the overall QoS of the network. In addition, these requirements do not explicitly take into account the heterogeneous data flow over the network. Since voice and data streams are sharing the same finite bandwidth, and data streams tend to contain much larger packets than VOIP, significant amounts of data can congest the network and prevent voice traffic from reaching its destination in a timely fashion. For this reason, most new hardware devices deployed on networks support QoS for VoIP [7]. 
VoIP deployments generally operate over various signalling and transport protocols which have a vital role in the management and transmission of the data packets, and also represent weak points and opportunities for malicious activities.

VOIP adds a number of complications to existing network technology, and these problems are magnified by security considerations. The packet switching nature of data networks allows multiple connections to share the same transport medium. Therefore, unlike telephones in circuit switched networks, an IP terminal endpoint can receive and potentially participate in multiple calls at once. Thus, an endpoint can be used to amplify attacks. On VoIP networks, resources such as bandwidth must be allocated efficiently and fairly to accommodate the maximum number of callers. This property can be violated by attackers who aggressively and abusively obtain an unnecessarily large amount of resources. Alternatively, the attacker simply can flood the network with large number of packets so that resources are unavailable to all other callers [5].

Theft of services and information is also problematic on VoIP networks. These threats are almost always due to active attack. Many of these attacks can be thwarted by implementing additional security controls at layer 2.This includes layer 2 security features such as DHCP Snooping [4].

Users may defer transitioning to IP Telephony if they believe it will reduce overall network security by creating new vulnerabilities that could be used to compromise nonVoIP systems and services within the same network.

Firewalls, network and system intrusion detection, authentication systems, antivirus scanners, and other security controls, which should already be in place, are required to counter attacks that might debilitate any or all IP-based services (including VoIP services).

The most comprehensive list of VoIP threats is maintained by VOIPSA at 8].

\section{VoIP Simulation Program Model (FCHAT).}

FCHAT is visual c++ socket programming package consists of two sides. The server side is play as a controller, authenticator and a communicator between the other clients. The client side is able to record, encrypt and send voice message to one or all clients. At the same time the client can receive, decrypted and replay the real time voice message. Fig. 1 describes how the server receive the connection request and if he accept the request then the "NEW user Address" is encrypted and broadcasted to all other clients. Fig. 2 describes the real time conversation between two clients through the server and how they can record, encrypt and send the messages in the same time that they can receive, decrypt and play the incoming messages. Fig.3 represents how client record, encrypt and send the messages to all other clients that are decrypt and replay the voice messages (conference). 
Figure (1): Broadcast authorized address

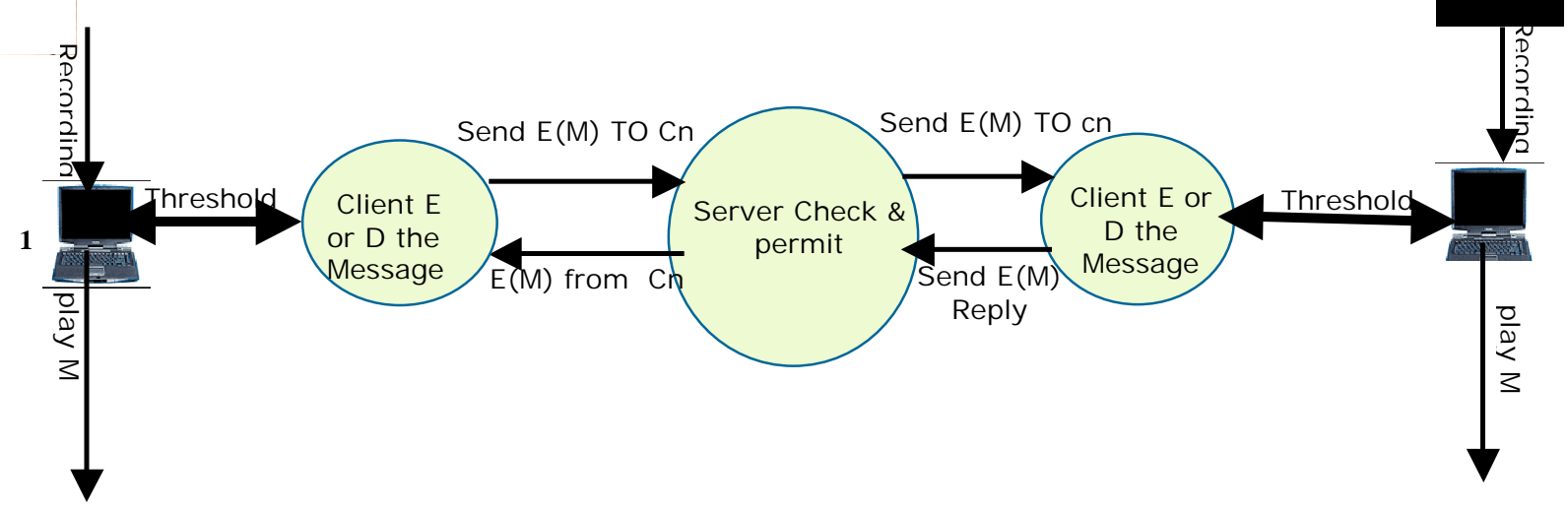

Figure (2): Real Time Conversation

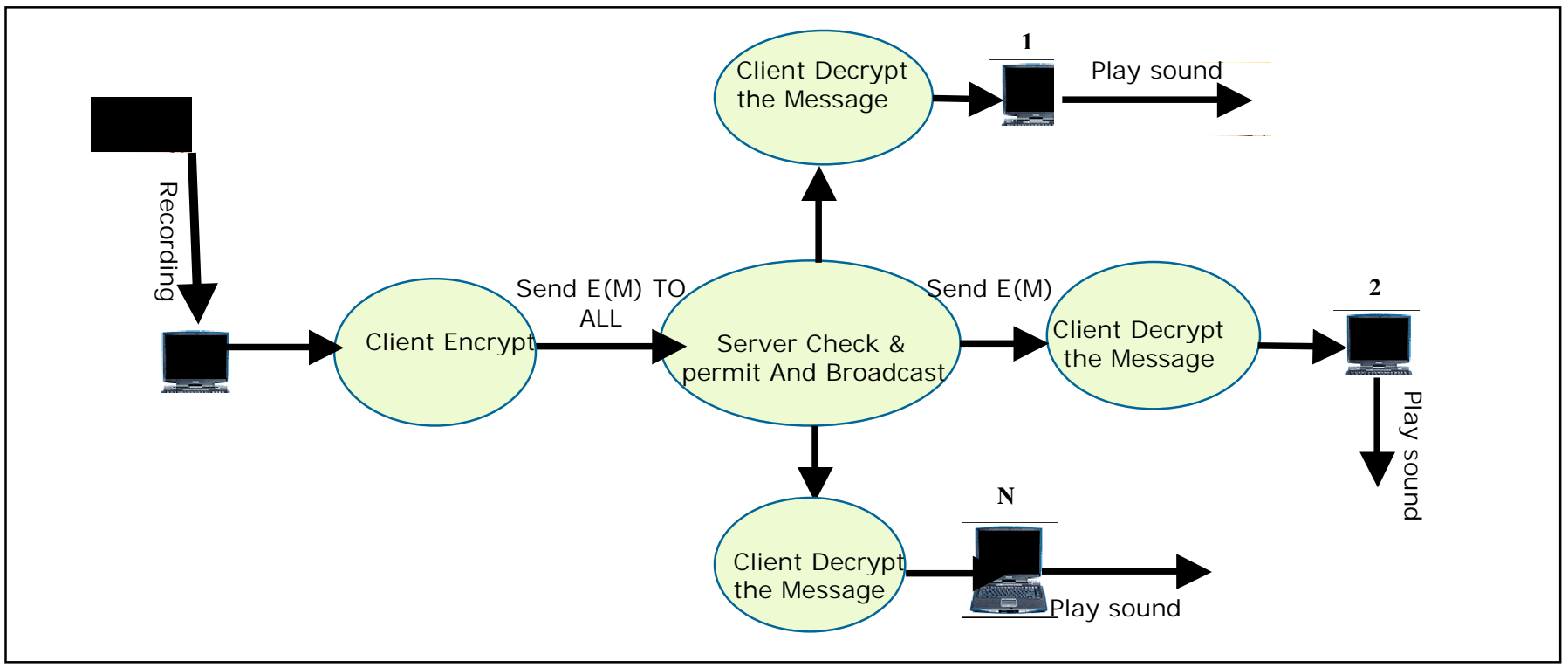

Figure (3): VoIP Conference 


\section{$\underline{3.1 \text { StsGui (NIST Statistical suite) }}$}

The National Institute of Standards and Technology (NIST) develop StsGui Test Suite as a statistical package consisting of 16 tests that were developed to test the randomness of (arbitrarily long) binary sequences produced by either hardware or software based Cryptographic random or pseudorandom number generators. These tests focus on a variety of different types of non randomness that could exist in a sequence. Some tests are decomposable into a variety of subtests. The 16 tests are mention in table 1.The test code was developed using a SUN workstation under the Solaris operating system. No guarantee is made regarding the compilation and execution of the PRNG implementations on other platforms. For this reason, a switch has been incorporated into the source codes to disable the inclusion of the PRNGs [9].

The objectives during the development of the NIST statistical test suite included:

- Platform Independence: The source code was written in ANSI C. However, some modification may have to be made, depending on the target platform and the compiler.

- Flexibility: The user may freely introduce their own math software routines.

- Extensibility: New statistical tests can easily be incorporated.

- Versatility: The test suite is useful in performing tests for PRNGs, RNGs and cipher algorithms. Portability: With minor modifications, source code may be ported to different platforms. The NIST source code was ported onto the SGI Origin, and a $200 \mathrm{MHz}$ PC using the Microsoft Visual C++ 6.0 development environment.

- Orthogonality: A diverse set of tests is provided.

- Efficiency: Linear time or space algorithms were utilized whenever possible.

The majority of the tests in the test suite either examine the distribution of zeroes and ones in some fashion, study the harmonics of the bit stream utilizing spectral methods, or attempt to detect patterns via some generalized pattern matching technique on the basis of probability theory or information theory.

In practice, any number of problems can arise if the user executes this software in unchartered domains. It is plausible that sequence lengths well beyond the testing procedure (i.e., on the order of 10) may be chosen. If memory is available, there should not be any reason why the software should fail. However, in many instances, user defined limits are prescribed for data structures and workspace [9].

- The parameter ALPHA denotes the significance level that determines the region of acceptance and rejection. NIST recommends that ALPHA be in the range $(0.001,0.01)$. 
Table (1): StsGui tests(From: [9])

\begin{tabular}{|c|l|}
\hline Test N0 & \multicolumn{1}{|c|}{ Description } \\
\hline 1. & The Frequency (Monobit) Test, \\
\hline 2. & Frequency Test within a Block, \\
\hline 3. & The Runs Test, \\
\hline 4. & Test for the Longest-Run-of-Ones in a Block, \\
\hline 5. & The Binary Matrix Rank Test, \\
\hline 6. & The Discrete Fourier Transform (Spectral) Test, \\
\hline 7. & The Non-overlapping Template Matching Test, \\
\hline 8. & The Overlapping Template Matching Test, \\
\hline 9. & Maurer's "Universal Statistical" Test, \\
\hline 10. & The Lempel-Ziv Compression Test, \\
\hline 11. & The Linear Complexity Test, \\
\hline 12. & The Serial Test, \\
\hline 13. & The Approximate Entropy Test, \\
\hline 14. & The Cumulative Sums (Cusums) Test, \\
\hline 15. & The Random Excursions Test, and \\
\hline 16. & The Random Excursions Variant Test. \\
\hline
\end{tabular}

\subsubsection{Modification and adaptation}

After the first compilation of the program suite the modification mentioned above appear to be needed. The system couldn't create the results folders and files, many library package were not include, need some adaptation with the operating system .Then we change the system setting to debugging mode then we begin to trace every code line and it's function then detect how the system calculate every parameter value(s) and discover many codes tricks and found the critical values for every test and by the way maintain the require adaptations. After this step and as the user manual advise [9] we run the tester using model file so as to be sure that modification does not change the logic of the program and the result is that :(the model pass all tests with P-value $=1$ ( $\max )$ for every test ; the model file name is data.e and it's report in appendix A. 


\subsection{Test bed environment}

Three PC computers. $(1.8 \mathrm{GHz})$ and one Lap Top ( $2 \mathrm{GHz})$ are used during the Experiments, the duration of one Experiment is approximately 30 Minutes, By using the model described in above section we were recorded a dialog in append mode file until the file size be greater than 13.2 MB. (Mandatory required). We save the recorded file as "DATAIN.TXT" which is used forty* times to be encrypted by Different Cryptographic Algorithms, and the outputs were saved in the files (d1.txt..d40.txt) which are converted to binary format so as to be enter one by one to StsGui (NIST Statistical Suite) package which was used to measure the randomness of each file. The output reports are summarized in the table 2. including the execution time of Encryption \& Decryption processes in seconds. Table 3. reorganized table 2 in a new form (so as to display the Experiments results and facts). Appendix A. summarized the P-values of sample tests.

\section{$\underline{\text { 4.Results and Discussions }}$}

- From table 3. Observed that all algorithms used ECB chain mode are fail to pass any randomness test. The BlowFish algorithm also doesn't pass all tests.

- XOR256BLOCK fail in 4 tests (fail in one test is enough to say it is not random).

- AES(CFB), TEA, and XOR256STREAM success in all tests, and to get which the optimum one special table of compression is constructed containing execution time (table 4). And for more clarify a graph representation for the three algorithms applied to (16 randomness tests) is constructed into Fig.4 (use table 5. P-values ).

- From Table 4. and Fig. 4. it is clear that TEA algorithm is the best one for VoIP encryption.

* Forty times due to change of the algorithm method or its situations (Padding, Chain). 


\section{Table (2): StsGui Experiments results}

\begin{tabular}{|c|c|c|c|c|c|c|}
\hline $\begin{array}{l}\text { FILE } \\
\text { NO }\end{array}$ & ALGO & Padding & Chain & Frequency & Number of Tests pass & $\begin{array}{l}\text { E.Time } \\
* *\end{array}$ \\
\hline B1 & $\operatorname{AES}(32)$ & ZEROS & ECB & 0.000000 & Zero & 11 \\
\hline B2 & $\operatorname{AES}(32)$ & BLANKS & $\mathrm{ECB}$ & 0.000000 & Zero & 11 \\
\hline B3 & $\operatorname{AES}(32)$ & PKCS7 & ECB & 0.000000 & Zero & 10 \\
\hline B4 & $\operatorname{AES}(16)$ & ZEROS & ECB & 0.000000 & Zero & 8 \\
\hline B5 & $\operatorname{AES}(24)$ & ZEROS & ECB & 0.000000 & Zero & 10 \\
\hline B6 & $\operatorname{AES}(32)$ & PKCS7 & $\mathrm{CBC}$ & 0.249284 & (15) *RUNS & 9 \\
\hline B7 & $\operatorname{AES}(32)$ & ZEROS & $\mathrm{CBC}$ & 0.249284 & (15) *RUNS & 9 \\
\hline B8 & $\operatorname{AES}(32)$ & BLANKS & $\mathrm{CBC}$ & 0.249284 & (15) *RUNS & 10 \\
\hline B9 & $\operatorname{AES}(32)$ & PKCS7 & CFB & 0.236810 & (16) & 9 \\
\hline $\mathrm{B} 10$ & $\operatorname{AES}(32)$ & ZEROS & CFB & 0.236810 & $(16)$ & 9 \\
\hline B11 & $\operatorname{AES}(32)$ & BLANKS & CFB & 0.236810 & (16) & 9 \\
\hline B12 & B.Fish & PKCS7 & ECB & 0.000000 & Zero & 14 \\
\hline B13 & B.Fish & ZEROS & ECB & 0.000000 & Zero & 14 \\
\hline B14 & B.Fish & BLANKS & ECB & 0.000000 & Zero & 14 \\
\hline B15 & B.Fish & PKCS7 & $\mathrm{CBC}$ & 0.000000 & Zero & 14 \\
\hline B16 & B.Fish & ZEROS & CFB & 0.000000 & Zero & 14 \\
\hline B17 & B.Fish & BLANKS & CFB & 0.000000 & Zero & 16 \\
\hline B18 & B.Fish & PKCS7 & CFB & 0.000000 & Zero & 15 \\
\hline B19 & TEA & ZEROS & ECB & 0.000000 & Zero & 10 \\
\hline B20 & TEA & BLANKS & ECB & 0.000000 & Zero & 10 \\
\hline B21 & TEA & PKCS7 & ECB & 0.000000 & Zero & 10 \\
\hline B22 & TEA & ZEROS & $\mathrm{CBC}$ & 0.657933 & (16) & 10 \\
\hline B23 & TEA & BLANKS & $\mathrm{CBC}$ & 0.657933 & $(16)$ & 10 \\
\hline B24 & TEA & PKCS7 & $\mathrm{CBC}$ & 0.657933 & (16) & 11 \\
\hline $\mathrm{B} 25$ & TEA & ZEROS & $\mathrm{CFB}$ & 0.699313 & (16) & 10 \\
\hline B26 & TEA & BLANKS & CFB & 0.699313 & $(16)$ & 10 \\
\hline B27 & TEA & PKCS7 & CFB & 0.699313 & (16) & 10 \\
\hline $\mathrm{B} 28$ & Xor256B & ZEROS & ECB & 0.000000 & Zero & 14 \\
\hline B29 & Xor256B & BLANKS & ECB & 0.000000 & Zero & 15 \\
\hline $\mathrm{B} 30$ & Xor256B & PKCS7 & ECB & 0.000000 & Zero & 15 \\
\hline B31 & Xor256B & ZEROS & $\mathrm{CBC}$ & 0.066882 & *C-sum,Fft,Apen,REV,Serial & 15 \\
\hline B32 & Xor256B & BLANKS & $\mathrm{CBC}$ & 0.066882 & ${ }^{*} \mathrm{C}$-sum,Fft,Apen,REV,Serial & 15 \\
\hline B33 & Xor256B & PKCS7 & $\mathrm{CBC}$ & 0.066882 & ${ }^{*} \mathrm{C}$-sum,Fft,Apen,REV,Serial & 15 \\
\hline B34 & Xor256B & ZEROS & $\mathrm{CFB}$ & 0.719747 & *Runs,Fft,Serial & 14 \\
\hline $\mathrm{B} 35$ & Xor256B & BLANKS & $\mathrm{CFB}$ & 0.719747 & *Runs,Fft,Serial & 14 \\
\hline B36 & Xor256B & PKCS7 & CFB & 0.719747 & *Runs,Fft,Serial & 13 \\
\hline B37 & Xor256S & & & 0.085587 & $(16)$ & 21 \\
\hline
\end{tabular}

** E.Time: Execution Time in seconds consumed to encrypt and decrypt (13.2 MByte) File

* :Fail in the following tests 
Proceedings of the $\boldsymbol{6}^{\text {th }}$ ICEENG Conference, 27-29 May, 2008

EE198 - 9

Table (3): Facts Summery

\begin{tabular}{|c|c|c|c|c|}
\hline FACT NO & EXPERMENTS & ALGORTHIM & CHAIN & FACT \\
\hline \multirow[t]{4}{*}{1} & B1..B3 & AES & \multirow{4}{*}{ ECB } & \multirow{4}{*}{ “0” TESTS } \\
\hline & B12..B14 & BLOWFISH & & \\
\hline & B19..B21 & TEA & & \\
\hline & B28..B30 & XOR256BLOCK & & \\
\hline \multirow[t]{3}{*}{2} & B12..B14 & \multirow{3}{*}{ BLOWFISH } & ECB & \multirow{3}{*}{ “0” TESTS } \\
\hline & B15 & & $\mathrm{CBC}$ & \\
\hline & B16..B18 & & CFB & \\
\hline \multirow[t]{3}{*}{3} & B28..B30 & \multirow{3}{*}{ XOR256BLOCK } & ECB & \multirow{3}{*}{ Pass “12" TESTS } \\
\hline & B31.. B33 & & $\mathrm{CBC}$ & \\
\hline & B34.. B36 & & CFB & \\
\hline 4 & B6..B8 & AES & $\mathrm{CBC}$ & Pass “15" TESTS \\
\hline 5 & B9..B11 & AES & CFB & Pass "all" TESTS \\
\hline \multirow[t]{2}{*}{6} & B22..B24 & \multirow[b]{2}{*}{ TEA } & $\mathrm{CBC}$ & \multirow[t]{2}{*}{ Pass “all” TESTS } \\
\hline & B25..B27 & & CFB & \\
\hline 7 & B37 & XOR256STREAM & & Pass “all" TESTS \\
\hline
\end{tabular}

Table 4: AES,TEA,XOR256S

\begin{tabular}{|c|c|c|c|c|}
\hline Algorithm & $\begin{array}{c}\text { Frequency } \\
\text { Test } \\
\text { Result }\end{array}$ & $\begin{array}{c}\text { Execution } \\
\text { Time } \\
13.2 \mathrm{Mb}\end{array}$ & $\begin{array}{c}\text { Execution Time for } \\
500 \mathrm{byte}\end{array}$ & Notes \\
\hline AES(CFB) & 0.236810 & $11 \mathrm{Sec}$ & $0.8 \mathrm{~m} \mathrm{sec}$ & \\
\hline TEA & 0.699313 & $10 \mathrm{Sec}$ & $0.72 \mathrm{~m} \mathrm{sec}$ & \\
\hline XOR256S & 0.085587 & $21 \mathrm{Sec}$ & $1.52 \mathrm{~m} \mathrm{sec}$ & \\
\hline
\end{tabular}


Proceedings of the $\boldsymbol{6}^{\text {th }}$ ICEENG Conference, 27-29 May, 2008

EE198 - 10

Table (5): XOR,AES,TEA Tests P-values of all tests

\begin{tabular}{|c|c|c|c|}
\hline Test No & XOR256S & AES & TEA \\
\hline 1 & 0.08856 & 0.23681 & 0.69931 \\
\hline 2 & 0.89776 & 0.11654 & 0.04011 \\
\hline 3 & 0.12962 & 0.30413 & 0.35049 \\
\hline 4 & 0.75976 & 0.77919 & 0.7749 \\
\hline 5 & 0.63712 & 0.86769 & 0.83292 \\
\hline 6 & 0.30413 & 0.01791 & 0.69931 \\
\hline 7 & 0.85138 & 0.35049 & 0.53415 \\
\hline 8 & 0.09658 & 0.79814 & 0.21331 \\
\hline 9 & 0.85138 & 0.83431 & 0.12962 \\
\hline 10 & 0.75976 & 0.22482 & 0.43727 \\
\hline 11 & 0.00576 & 0.77919 & 0.88317 \\
\hline 12 & 0.58521 & 0.31154 & 0.23276 \\
\hline 13 & 0.68902 & 0.00316 & 0.60246 \\
\hline 14 & 0.69931 & 0.24928 & 0.59555 \\
\hline 15 & 0.18156 & 0.28967 & 0.59555 \\
\hline
\end{tabular}

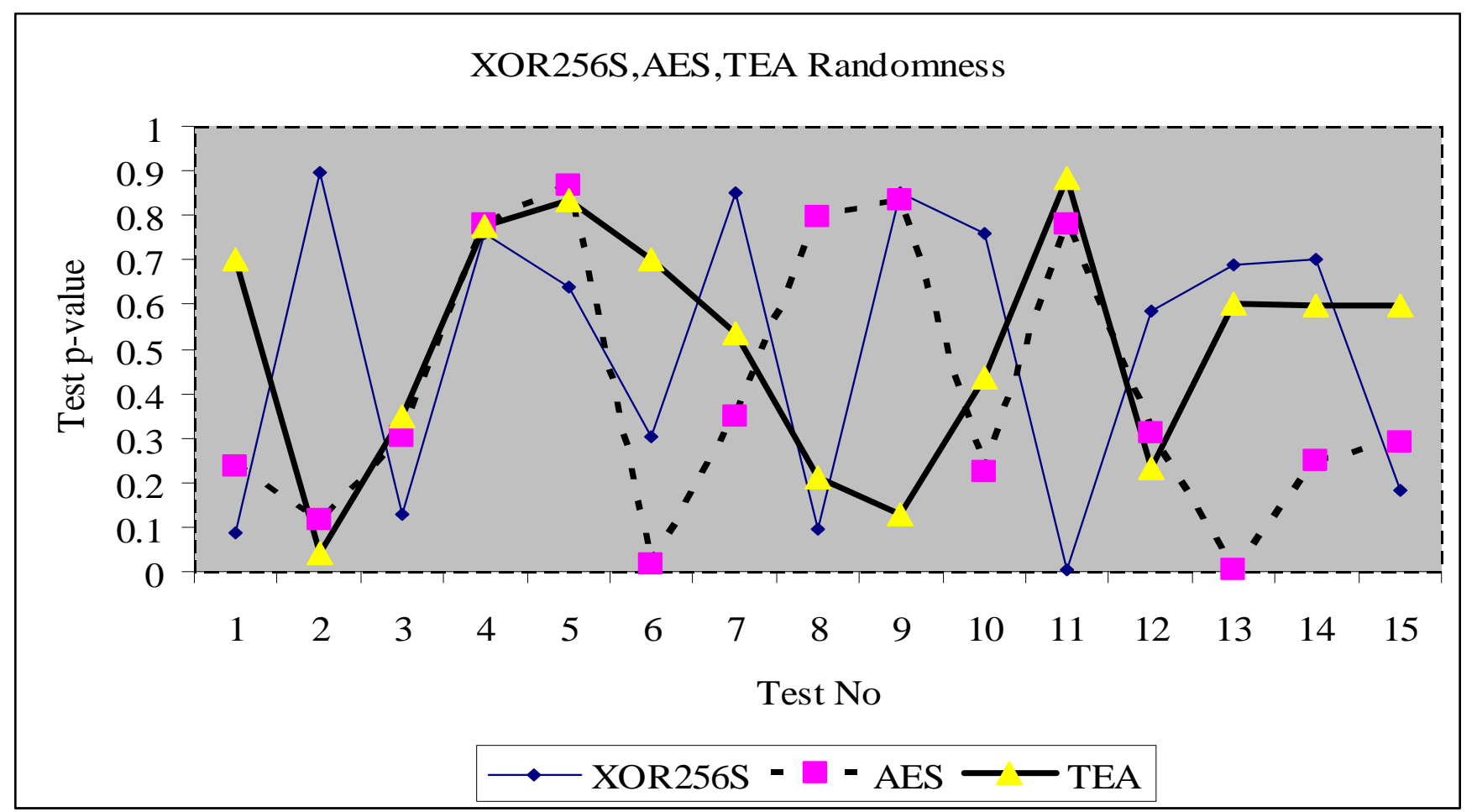

Figure (4): XOR,AES,TEA Tests 


\section{Conclusions:}

This paper conclude that the TEA algorithm is more suitable to encrypt VoIP due to its encryption strength (more random) and it's smallest execution time which minimize the total end to end delay and has the least negative effect on QoS.

\section{$\underline{\text { References: }}$}

[1] Praphul Chandra andLide, Wi-Fi TelephonyChallenges and Solutionsfor Voice over WLANs, AMSTERDAM • BOSTON • HEIDELBERG • LONDON, 2007.

[2] Amarandei-Stavila Mihai Voice over IP Security A layered approach, AmarandeiStavila Mihai mihaia@gmail.com, XMCO consultants.

[3] L. Sun, SPEECH QUALITY PREDICTION FOR VOICE OVER INTERNET PROTOCOL NETWORKS, Ph.D. January 2004.

[4] An ISS Whitepaper, VoIP: The Evolving Solution and the Evolving Threat, MCVOIPWP-1204,2004.

[5] Thomas Porter, PracticalVoIP Security, Syngress, 2007.

[6] Juniper Networks, Inc., Voice Over IP 101, www.juniper.net, Part Number: 200087-002 May 2007.

[7] Steven Sullivan,Securing a Converged Network

[8] D. Richard Kuhn, Thomas J. Walsh, Steffen Fries , Security Considerations for Voice Over IP Systems, www.voipsa.com/Activities/taxonomy.php,NISTSP 80058, January 2005 .

[9] Andrew Rukhin, Juan Soto, James Nechvatal,Miles Smid, Elaine Barker, Stefan Leigh,Mark Levenson, Mark Vangel, David Banks,Alan Heckert, James Dray, San Vo, A STATIISTIICAL TEST SUIITE FOR RANDOM AND PSEUDORANDOM NUMBER GENERATORS FOR CRYPTOGRAPHIIC APPLIICATIIONS, NIST Special Publication 800-22(with revision dated December 2000).

[10] A. Elbayoumy,S. Shepherd, A high grade secure VoIP system using the tiny encryption algorithm, Proceeding of 7 th Annual International Symposium on advanced Radio Technologies Boulder, Colerader,1-3 March 2005 . 
Proceedings of the $\boldsymbol{6}^{\text {th }}$ ICEENG Conference, 27-29 May, 2008

EE198 - 12

\section{Appendix A. summarized the P-values of sample tests}

\begin{tabular}{|c|c|ccc|c|c|c|c|c|c|}
\hline $\begin{array}{c}\text { STATISTICAL } \\
\text { TEST }\end{array}$ & $\begin{array}{c}\text { PALUE } \\
\text { model } \\
\text { file }\end{array}$ & $\begin{array}{c}\text { VALUE } \\
\text { fail file }\end{array}$ & $\begin{array}{c}\text { VALUE } \\
\text { B6 }\end{array}$ & $\begin{array}{c}\text { VALUE } \\
\text { B9 }\end{array}$ & $\begin{array}{c}\text { VALUE } \\
\text { B22 }\end{array}$ & $\begin{array}{c}\text { P- } \\
\text { VALUE } \\
\text { B25 }\end{array}$ & $\begin{array}{c}\text { PA- } \\
\text { VALUE } \\
\text { B31 }\end{array}$ & $\begin{array}{c}\text { P- } \\
\text { VALUE } \\
\text { B34 }\end{array}$ & $\begin{array}{c}\text { P- } \\
\text { VALUE } \\
\text { B37 }\end{array}$ \\
\hline $\begin{array}{c}\text { frequency } \\
\text { block- } \\
\text { frequency }\end{array}$ & 1 & 0 & 0.249284 & 0.23681 & 0.65793 & 0.69931 & 0.06688 & 0.71975 & 0.08856 \\
\hline $\begin{array}{c}\text { cumulative- } \\
\text { sums }\end{array}$ & 1 & 0 & 0.032923 & 0.11654 & 0.36692 & 0.04011 & 0.53415 & 0.65793 & 0.89776 \\
\hline runs & 1 & 0 & 0.051942 & 0.77919 & 0.5749 & 0.7749 & 0.23681 & 0.08559 & 0.75976 \\
\hline longest-run & 1 & 0 & 0.911413 & 0.86769 & 0.41902 & 0.83292 & 0.22482 & 0.0156 & 0.63712 \\
\hline rank & 1 & 0 & 0.911413 & 0.01791 & 0.20227 & 0.69931 & 0.88317 & 0.75976 & 0.30413 \\
\hline fft & 1 & 0 & 0.202268 & 0.35049 & 0.61631 & 0.53415 & 0.00056 & $3.7 \mathrm{E}-05$ & 0.85138 \\
\hline $\begin{array}{c}\text { nonperiodic- } \\
\text { templates }\end{array}$ & 1 & 0 & 0.798139 & 0.79814 & 0.61631 & 0.21331 & 0.23681 & 0.61631 & 0.09658 \\
\hline $\begin{array}{c}\text { overlapping- } \\
\text { templates }\end{array}$ & 1 & 0 & 0.816537 & 0.83431 & 0.22482 & 0.12962 & 0.53415 & 0.38383 & 0.85138 \\
\hline universal & 1 & 0 & 0.090936 & 0.22482 & 0.12962 & 0.43727 & 0.61631 & 0.21331 & 0.75976 \\
\hline apen & 1 & 0 & 0.55442 & 0.77919 & 0.88317 & 0.88317 & 0.14533 & 0.28967 & 0.00576 \\
\hline $\begin{array}{c}\text { random- } \\
\text { excursions }\end{array}$ & 1 & 0.392456 & 0.77276 & 0.31154 & 0.63712 & 0.23276 & 0.63712 & 0.4528 & 0.58521 \\
\hline $\begin{array}{c}\text { random- } \\
\text { excursions- } \\
\text { variant }\end{array}$ & 1 & 1.392456 & 0.090936 & 0.00316 & 0.4686 & 0.60246 & 0.04281 & 0.31154 & 0.68902 \\
\hline $\begin{array}{c}\text { serial } \\
\text { linear- } \\
\text { complexity }\end{array}$ & 1 & 1 & 0 & 0.678686 & 0.28967 & 0.17187 & 0.59555 & 0.97807 & 0.9717 & 0.18156 \\
\hline
\end{tabular}

